\title{
Intraoperative Monopolar Radiofrequency Ablation in Chronic Atrial Flutter with Concomitant Pulmonary Stenosis
}

\author{
Ichiro Kashima*, Yoshito Inoue, Kenichi Hashizume and Ryuichi Takahashi \\ Department of Cardiovascular Surgery, Saiseikai Utsumomiya Hospital, Utsunomiya, Tochigi, Japan
}

\begin{abstract}
We successfully treated a 54 year old woman suffering pulmonary stenosis with chronic and drug resistant atrial flutter by means of intraoperative radiofrequency ablation. Ablation applied to the cavotricuspid isthmus by a visible monopolar electrode is easy to perform as a concomitant procedure during repair of congenital structural heart disease.
\end{abstract}

Keywords: Intraoperative radiofrequency ablation, congenital structural heart disease, atrial flutter, pulmonary stenosis.

\section{INTRODUCTION}

Atrial flutter (AF) is a life-threatening tachycardia seen in long-term survivors of congenital structural heart disease (CSHD) and leads to left atrial dilatation and atrial fibrillation [1]. Supraventricular arrhythmia in CSHD generally develops a long time after surgery, because the atriotomy scar is one of its causes; definitive repair tends to take place only in early infancy. Medication and/or catheter ablation are therefore often more feasible than surgical intervention [2]. In the present case, we put an end to chronic and drug resistant $\mathrm{AF}$ by intraoperative radiofrequency ablation (RFA) of the cavotricuspid isthmus (CTI) during surgical repair of pulmonary stenosis.

\section{CASE}

A 54-year-old woman had been diagnosed with pulmonary stenosis as a high school student, but she left it untreated until she found recent dyspnea upon making effort. Chest radiography showed moderate cardiomegaly with a $60 \%$ cardio-thoracic ratio, and electrocardiography (ECG) showed common AF with a typical 'sawtooth' pattern and inverted $p$ waves in leads II, III, and $\mathrm{aV}_{\mathrm{F}}$. Her atrial frequency was $280 /$ min with $2: 1$ or $4: 1$ conduction block. Doppler echocardiography revealed mild tricuspid regurgitation, together with a high right ventricular systolic pressure of $85 \mathrm{mmHg}$ at the tricuspid valve (TV) level, pulmonary and infundibular stenosis, and a hypertrophic right ventricle. She was initially prescribed propafenone with coumadin as an antiarrhythmic therapy, but her AF remained. Following rate control by beta blockers, she underwent elective surgical repair for obstruction of the right ventricular outflow tract (RVOT). The operation involved median sternotomy with extracorporeal circulation under general anesthesia. Following cardioplegic arrest, an oblique atrial incision was made, and the CTI was ablated linearly using a monopolar RFA system (Cobra ESU, Boston Scientific-EP Technologies, Natick, MA) (Fig. 1). This procedure took only a few minutes. The main pulmonary

*Address correspondence to this author at the Department of Cardiovascular Surgery, Saiseikai Utsunomiya Hospital, 911-1, Takebayashi-machi, Utsunomiya, Tochigi, 321-0974 Japan;

E-mail: i.kashima@ashikaga.jrc.or.jp artery was then opened, and the incision was extended to the RVOT with pulmonary valvotomy. After division of the parietal muscle bundles, the RVOT was remade using an equine pericardial transannular patch (Xenomedica, Baxter Healthcare Co., Deerfield, IL). At the end of the procedure, her heart immediately resumed in a spontaneous sinus rhythm. The duration of cardiopulmonary bypass was 67 minutes, and aortic cross clamping lasted 57 minutes. The postoperative course was good, and sinus rhythm was maintained throughout her time in hospital prior to discharge. Postoperative echo-Doppler showed significant reduction of right ventricular pressure, to $18 \mathrm{mmHg}$ at the TV level, and showed mild tricuspid and pulmonary regurgitation. She was in class I of the New York Heart Association classification, and ECG showed the presence of regular sinus rhythm. This was followed up by Holter ECG, which confirmed no recurrence of AF six years after the operation.

\section{DISCUSSION}

$\mathrm{AF}$ is considered to be caused by a reentry circuit that develops between two electrical block lines along the crista terminalis and tricuspid annulus, respectively the posterior barrier and anterior barrier [3]. Formation of the posterior barrier is probably related to tissue abnormality associated with atrial dilatation, aging, atriotomy scar or other factors [2]. It is generally accepted that treatment for AF is desirable prior to conversion to atrial fibrillation, and that ablation of the CTI is a good remedy for AF, even in the presence of CSHD $[1,4,5]$. Ablation by catheter may be more difficult in the presence of structural heart disease [6]. Intraoperative cryoablation (CA) of the CTI has been reported as effective against chronic $\mathrm{AF}$ in patients with residual pulmonary stenosis of tetralogy of Fallot [5]. It is commonly believed that the efficacy of RFA is the same as that of CA [7]. The CTI is too thick for the bipolar electrode with the RFA system, however. The advantage of using the Cobra ESU monopolar RFA system is in rapidly setting up a linear conduction block, because of its seven flexible monopolar electrodes. We believe that the monopolar RFA system applied to CTI under direct vision is an easy and curative technique for typical AF. 
In conclusion, intraoperative monopolar RFA applied to the CTI is a good option for chronic AF in candidates for surgery who have CSHD.

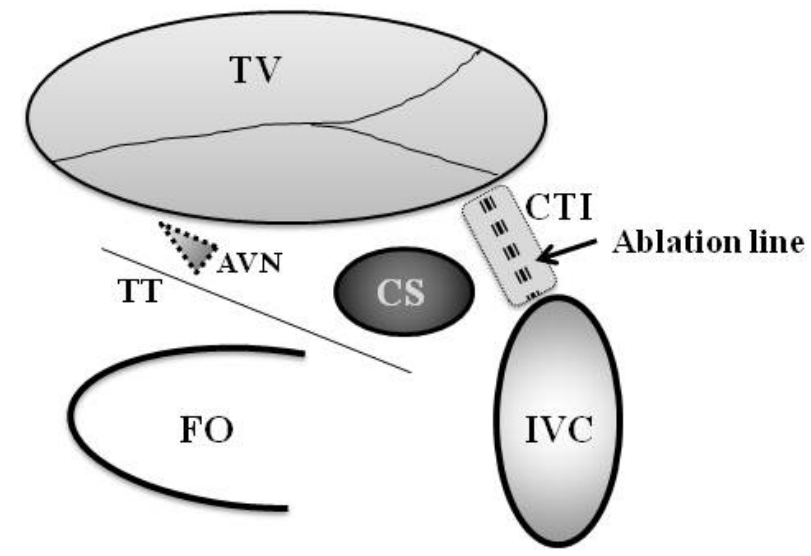

Fig. (1). Internal structures of the right atrium. The CTI lies between the orifice of the IVC and the attachment of the septal leaflet of the TV. AVN: Atrioventricular node, CS: Coronary sinus, CTI: cavotricuspid isthmus, FO: Fossa ovalis, IVC: Inferior vena cava, TT: Tendon of Todaro, TV: Tricuspid valve.

\section{REFERENCES}

[1] Li W, Somerville J. Atrial flutter in grown-up congenital heart (GUCH) patients clinical characterisitics of affected population. Int J Cardiol 2000; 75: 129-37.

[2] Chan DP, Van Hare GF, Mackall JA, Carlson MD, Waldo AL. Importance of atrial flutter isthmus in postoperative intra-atrial reentrant tachycardia. Circulation 2000; 102: 1283-9.

[3] Olgin JE, Kalman JM, Fitzpatrick AP, Lesh MD. Role of right atrial endocardial structures as barriers to conduction during human type I atrial flutter. Circulation 1995; 92: 1839-48.

[4] Lickfett L, Calkins $\mathrm{H}$, Nasir K, et al. Clinical prediction of cavotricuspid isthmus dependence in patients referred for catheter ablation of "typical" atrial flutter. J Cardiovasc Electrophysiol 2005; 16: 969-73.

[5] Therrien J, Harris L, Dore A, et al. Impact of pulmonary valve replacement on arrhythmia propensity late after repair of tetralogy of Fallot. Circulation 2001; 103: 2489-94.

[6] Calkins H, EI-Atassi R, Kalbfleisch SJ, Langberg JJ, Morady F. Catheter ablation of atrial flutter using radiofrequency energy. Circulation 1992; 86: I-723.

[7] Timmermans C, Ayers GM, Crijns HJGM, Rodriguez LM. Randomized study comparing radiofrequency ablation with cryoablation for the treatment of atrial flutter with emphasis on pain perception. Circulation 2003; 107: 1250-2. 